Ensino

\title{
MHS e MCU: a conexão desses movimentos no ensino de física
}

\author{
SHM and UCM: the connection of these movements in the teaching of \\ physics
}

\section{Rafaela Coutinho de Oliveira'(i), Édino da Silva Brito'(D), Gabriel Willian Callegari'(1), Alexandre Jitsuo Fuzita' ${ }^{\circledR}$, Vitor Hugo Dias Ferrara' ${ }^{\circledR}$, Daniela Lopes Elizeu'(i), Saulo Portes dos Reis ${ }^{1}$ (i)}

\author{
' Instituto Federal de São Paulo, Votuporanga, SP, Brasil
}

\section{RESUMO}

O presente trabalho é o resultado de um esforço conjunto realizado entre alunos da disciplina Oscilações e Ondas do curso de licenciatura em física do Instituto Federal de São Paulo. Diante das inúmeras equações diferenciais inerentes ao estudo do movimento oscilatório, surgiu a questão: como abordar esse tema no ensino médio? A dificuldade reside no fato de que o aluno nessa etapa de ensino não tem como compreender equações diferenciais. Sendo assim, foi desenvolvido uma proposta de abordagem para o ensino do Movimento Harmônico Simples (MHS), a partir da relação desse movimento com o Movimento Circular Uniforme (MCU). Além disso, propõe-se uma demonstração para as equações dos períodos desse tipo de movimento. Com o intuito de trazer ao aluno uma experiência mais concreta acerca do tema, dois experimentos com material de baixo custo foram propostos e executados pelos autores.

Palavras-chave: movimento harmônico; movimento circular; ensino de física;

\section{ABSTRACT}

The present work is the result of a joint effort carried out among students of the discipline Oscilações e Ondas of the Physics degree course at the Federal Institute of São Paulo. In view of the numerous differential equations inherent to the study of oscillatory movement, the question arose: how to approach this topic in high school? The difficulty lies in the fact that the student at this stage of teaching has no way of understanding differential equations. Therefore, a proposal for an approach to teaching Simple Harmonic Movement (SHM) was developed, based on the relationship of this movement with the Uniform Circular Movement (UCM). In addition, we propose a demonstration for the equations of the periods of this type of movement. In order to bring the student a more concrete experience on the subject, two experiments with low-cost material were proposed and executed by the authors.

Keywords: harmonic movement; circular movement; physics teaching; 


\section{INTRODUÇÃO}

É um consenso entre professores que está cada vez mais difícil motivar os alunos nas aulas de física (ver [9]). Esse quadro pode ser ainda mais desfavorável no caso de conteúdos não tão evidenciados na estrutura curricular, como o conceito de "Oscilações" (ver [6]). Mais ainda, conteúdos nos quais as abordagens se restringem a apresentação de equações prontas e resoluções de exercícios, trazem para o aluno a ideia de que a disciplina de física é extremamente difícil (ver [3]). No caso do tema de Oscilações, alguns trabalhos utilizam de softwares (ver [2] e [9]) com o intuito de tornar o conteúdo mais acessível, entretanto, esses trabalhos são destinados a alunos do ensino superior, uma vez que utilizam o softwares que necessitam da compreensão de equações diferenciais para serem utilizados.

Ora, os fenômenos oscilatórios estão presentes em diversas formas na natureza, e seu estudo e entendimento colabora para o desenvolvimento em várias áreas do conhecimento, tais como, engenharia, química, biologia, etc, além de ser encontrada em praticamente todos os campos da física (ver [10]). Nos cursos de ensino superior, o conteúdo de oscilações é devidamente abordado com o auxílio de equações diferenciais ordinárias de segunda ordem. Lá o aluno aprende que uma equação do tipo

$$
\frac{d^{2}}{d t^{2}} x(t)+\omega^{2} x(t)=0
$$

descreve um movimento harmônico simples com frequência de vibração $f=\frac{\omega}{2 \pi}$ e período $T=\frac{2 \pi}{\omega}$, ao longo do eixo x. Na equação 1 acima, $\omega$ é chamado de frequência angular de oscilação do sistema, sendo que seu valor depende apenas das variáveis dinâmicas do sistema.

A equação 1 pode ser facilmente obtida a partir da segunda lei de Newton, possibilitando assim ao aluno calcular de maneira direta o valor de $\omega$. Aqui ressaltamos que o presente trabalho se detém ao estudo do sistema massa-mola e do pêndulo simples, dessa forma, para esses casos os valores das frequências $\omega_{m m}$ e $\omega_{p s}$ dos sistemas massa-mola e pêndulo simples, são respectivamente: 


$$
\omega_{m m}=\sqrt{\frac{k}{m}} \text { e } \omega_{p s}=\sqrt{\frac{\ell}{g}} .
$$

Em se tratando de ensino, acreditamos que as equações das frequências angulares apresentadas acima, apresentam um grande potencial em termos motivacionais. Esse potencial se deve ao fato de que experimentos simples, feitos com materiais de baixo custo, podem ser preparados afim de se obter os parâmetros descritos por essas equações, sem a necessidade do uso de softwares. Note que, para obter o valor da gravidade local, basta apenas obter o período de oscilação de um pêndulo, da mesma forma, pode-se obter o valor de uma massa desconhecida utilizando um cronômetro e um sistema massa-mola.

Entretanto, algumas questões surgem naturalmente para o futuro licenciado em física: como ensinar movimento harmônico simples para o ensino médio? Como explicar os cálculos do período e da frequência de oscilação de um oscilador harmônico simples? Toda essa dificuldade se deve ao fato de que alunos do ensino médio não tem contato com cálculo diferencial, dessa forma, se torna impossível a obtenção, bem como a compreensão, das equações apresentadas em 1 via equações diferenciais.

Diante dessas dificuldades, as quais foram apresentadas pelos licenciandos do curso de física do Instituto Federal de São Paulo (Votuporanga), propomos uma abordagem do ensino do movimento harmônico simples (aqui descrito por MHS) para alunos do ensino médio que possibilite a obtenção das equações apresentadas em 2 sem o uso de equações diferenciais.

Entendemos que a importância de uma demonstração formal dessas equações se deve ao fato de poderem ser confrontadas com experimentos simples, os quais podem ser realizados pelos próprios alunos do ensino médio. A proposta se resume a descrever o MHS a partir das equações do movimento circular uniforme (MCU), uma vez que, o MHS pode ser entendido como a projeção de um MCU ao longo do eixo x, conforme discutido adiante. Cabe ressaltar que alguns textos do ensino superior fazem essa abordagem, por exemplo, em [8] temos uma breve descrição dessa relação, enquanto que uma descrição mais elaborada é apresentada em [12]. Todavia, em nenhum desses textos a relação entre as frequências angulares e os parâmetros dinâmicos do movimento (equações 2) é 
demonstrada sem o uso das equações diferenciais. Cabe destacar ainda que nem mesmo livros textos muito utilizados nos cursos superiores sequer fazem menção a essa relação, aqui citamos por exemplo o ótimo livro [5], e o mundialmente conhecido [4].

Dessa forma, nossa proposta tem como ponto de partida a compreensão da matemática que nos permite obter as relações entre as frequências angulares e os parâmetros dinâmicos do sistema. Após, propomos dois experimentos simples para serem feitos, afim de se obter o valor do campo gravitacional local e de uma massa m, supostamente desconhecida. Acreditamos que a realização desses experimentos irá colaborar em uma melhor compreensão do aluno acerca dos conceitos inerentes ao MHS: período, frequência e frequência angular.

\section{DESCRIÇÃO TEÓRICA}

\subsection{Introdução}

Para compreender a proposta do presente artigo, uma introdução teórica se faz necessária. Dessa forma, consideramos de extrema importância começar com a definição do conceito oscilação, o qual está intimamente ligado a ideia de equilíbrio estável. Nossa percepção intuitiva associa a palavra oscilação a repetição: um pêndulo oscila de uma extremidade a outra, uma massa preza a uma mola também pode oscilar.

Uma oscilação sempre surge a partir da perturbação de um sistema em equilíbrio estável. Um sistema de equilíbrio estável é aquele em que, para pequenas perturbações, o corpo tende a retornar para a posição de equilíbrio, novamente o sistema massa-mola e o pêndulo simples são exemplos canônicos. Quando há perturbação a um sistema de equilíbrio instável, o corpo não tende a voltar para a posição de equilíbrio, assim, não haverá um movimento oscilatório.

Portanto, pode-se concluir: "uma oscilação é a resposta da natureza a uma perturbação de um sistema de equilíbrio estável". O quão "rápido" será essa oscilação dependerá apenas das variáveis dinâmicas do problema, as quais serão determinadas matematicamente com auxílio do MCU. Mais ainda, deve-se levar em conta que a 
oscilação ocorrerá apenas para "pequenas" perturbações em torno da posição de equilíbrio, sendo que cabe a avaliação do quão pequeno deve ser essa perturbação cabe a quem estiver analisando o problema. A figura 1 ilustra os dois tipos de equilíbrio.

Figura 1 - Ilustração dos dois tipos de equilíbrio

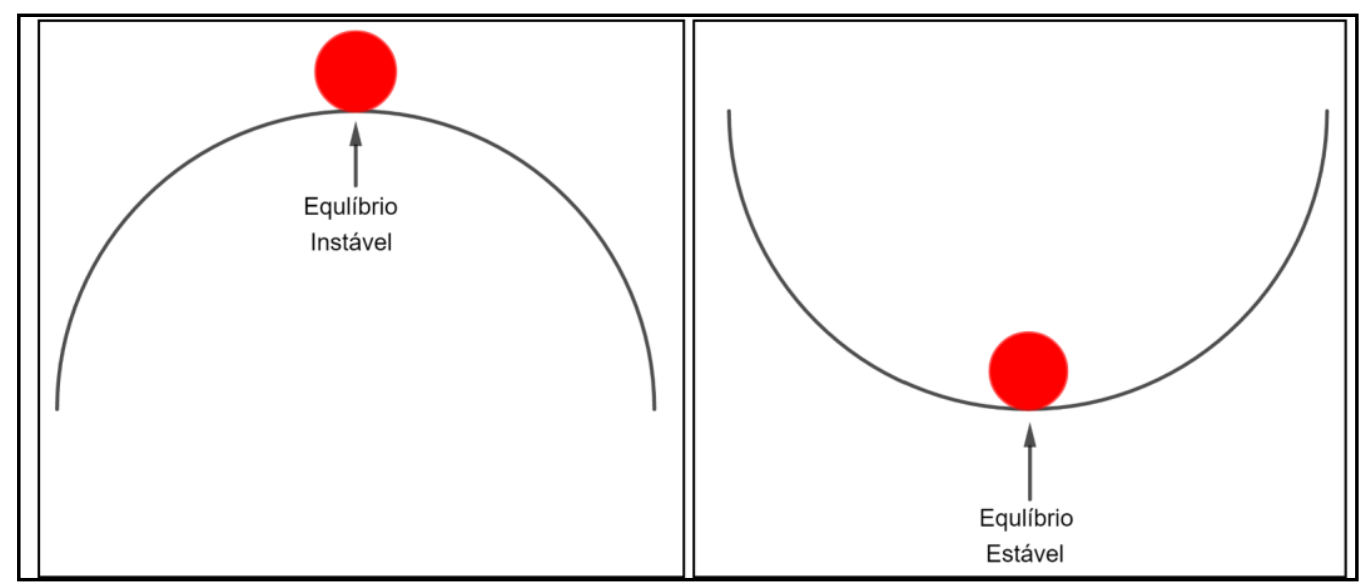

Fonte: Autores (2021)

\subsection{Algumas definições}

Essa seção destina-se a apresentar formalmente a definição de alguns termos utilizados no MHS.

Amplitude é a máxima "distância" que o sistema se encontra da posição de equilíbrio;

Período é o tempo necessário para realizar uma oscilação completa;

Frequência é o inverso do período, isto é, é a quantidade de oscilações executadas por unidade de tempo. Denotando o período por T e a frequência por $f$ tem-se:

Número de oscilações
Tempo necessário

$\mathrm{T}$

$1 \mathrm{~s}$

Fazendo-se a regra de três, têm-se: 


$$
f=\frac{1}{T}
$$

Os termos período e frequência já eram utilizados no estudo do movimento circular uniforme (MCU), naquele contexto, o período é o tempo necessário para o sistema completar uma volta e a frequência é o número de voltas completadas em um intervalo de tempo. A analogia com o MHS é imediata.

No MCU havia ainda a velocidade angular, a qual se referia ao ângulo percorrido por unidade de tempo. A velocidade angular do MCU pode também ser associada ao MHS, porém, o termo mais adequado passa a ser frequência angular. A analogia é feita da seguinte forma, a cada oscilação completa, associa-se o número $2 \pi$. Por exemplo, se o sistema oscila com uma frequência de $1 \mathrm{~Hz}$, ou seja, uma oscilação por segundo, diz-se que sua frequência angular é de $2 \pi \mathrm{Hz}$. É importante compreender que não há nenhum ângulo a ser percorrido, é uma simples associação, a qual ficará completamente justificada na próxima seção. Denotando a frequência angular por $\omega$ (o mesmo símbolo na equação 1), tem-se:

$$
\omega=2 \pi f \text {. }
$$

\subsection{Relação entre MHS e MCU}

\subsubsection{Introdução}

Conforme exposto anteriormente, a ideia de se associar o MCU ao MHS se deve ao fato de que qualquer movimento oscilatório pode ser entendido como a projeção, no eixo correspondente, de um movimento circular. Imagine, por exemplo, um sistema executando um movimento oscilatório de amplitude dada por $A$ no eixo $x$. Cada posição marcada no eixo $x$ pode ser entendida como sendo a projeção (nesse mesmo eixo) de um movimento circular uniforme sendo realizado em uma 
circunferência cujo raio vale $A$, ou seja, o raio da circunferência equivale a amplitude do movimento oscilatório.

Para elucidar melhor essa analogia apresenta-se a sequência na figura 2. Perceba que o ponto azul pode ser compreendido como sendo à projeção do ponto vermelho ao longo do eixo x. Dessa forma, a medida que o ponto vermelho descreve um MCU, o ponto azul descreve um MHS. Note ainda que quando o corpo azul oscila da posição $A$ para a posição $-A$ no eixo $x$, o movimento descrito pelo ponto vermelho realiza meia meia volta na parte superior da circunferência, isto é, no sentido antihorário. Já o movimento oscilatório da volta, isto é, quando o ponto azul vai de $-A$ até $A$, o movimento circular correspondente percorre a semi-circunferência inferior. Dessa forma, uma oscilação completa no eixo x pode ser associada a uma volta completa no movimento circular.

Figura 2 - O movimento oscilatório corresponde ao movimento da esfera azul, já a esfera vermelha descreve o movimento circular associado

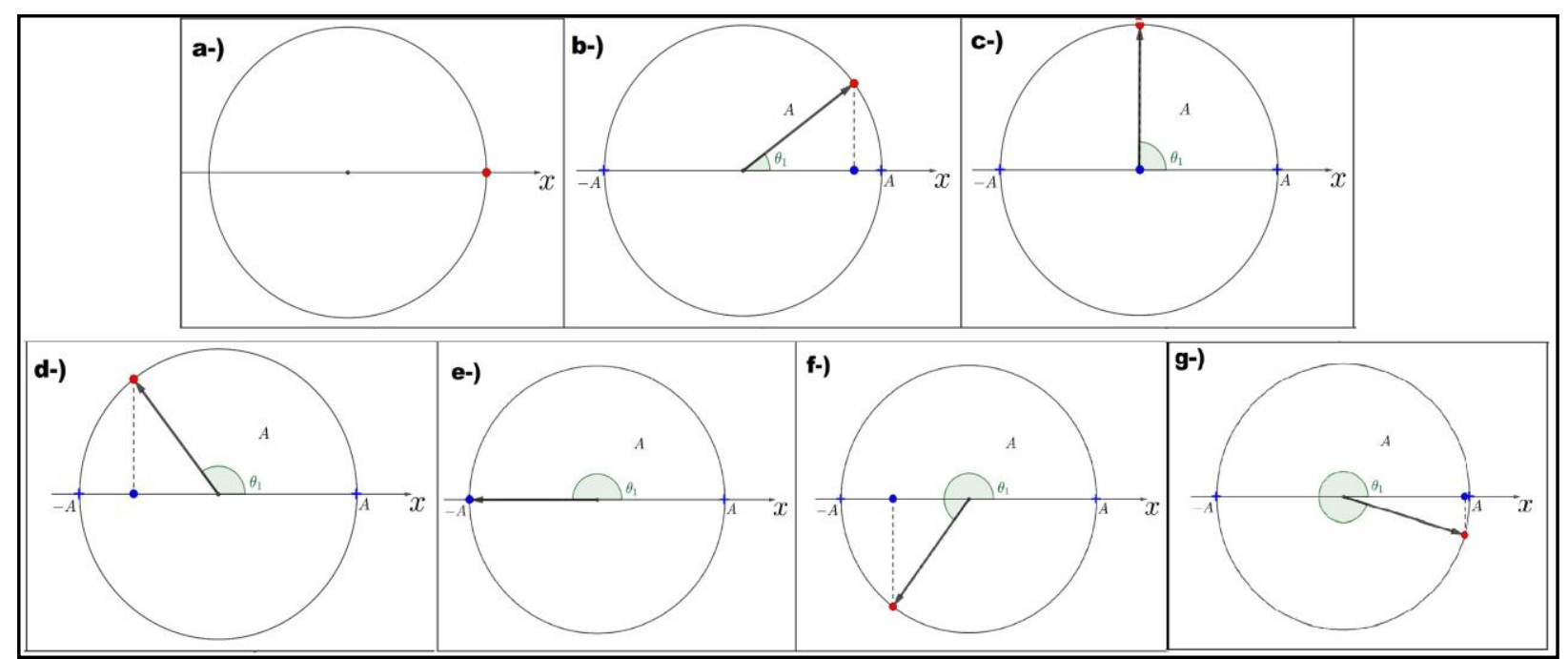

Fonte: Autores (2021)

Em que: a-) Início do movimento oscilatório na amplitude A. O movimento circular correspondente está associado ao arco 0. b-) A partícula oscilante se desloca em direção à posição de equilíbrio, aumentando o módulo de sua velocidade. A partícula no movimento circular associado mantém o módulo de sua velocidade constante, porém, nesse momento descreveu um arco $\theta_{1}$. Assim, a cada posição no eixo $x$, associa-se um arco de circunferência. c-) A partícula oscilante se encontra na posição de equilíbrio, o arco associado a essa posição é $\frac{\pi}{2}$. d-) A partícula oscilante passou a posição de equilíbrio, agora o módulo de sua velocidade passa a diminuir e o arco associado está entre $\frac{\pi}{2}$ e $\pi$. e-) A partícula oscilante atinge a posição de amplitude $-\mathrm{A}$, o arco associado vale $\pi$. f-) A partícula oscilante inicia o movimento de volta, novamente aumentando o módulo de sua velocidade, o arco associado está entre $\pi \mathrm{e} \frac{3 \pi}{2}$. g-) A partícula oscilante está prestes a completar a oscilação, assim como a partícula em movimento circular está completando uma volta, isto é, o movimento circular irá completar um arco igual a $2 \pi$. 
Diante do exposto acima, pode-se concluir que a frequência do movimento oscilatório é a mesma frequência do movimento circular associado, isto é, o número de oscilações realizados em cada unidade de tempo é igual ao número de voltas completas na circunferência por unidade de tempo.

As três grandezas apresentadas (período, frequência e frequência angular) estão correlacionadas, assim basta conhecer uma delas e se identifica as outras duas. A identificação é facilitada pensando no movimento circular associado. Uma vez que os parâmetros de um movimento harmônico podem ser obtidos com relação a um movimento circular associado, as equações do movimento também devem ser.

\subsection{Determinando as equações do movimento}

A segunda lei de Newton nos indica que para descrever completamente qualquer movimento de um corpo, isto é, para obter a equação horária dos espaços, necessita-se conhecer todas as forças que atuam no corpo. Entretanto, apenas o conhecimento das forças não é suficiente para escrever a equação horária. Imaginemos por exemplo que um corpo sujeito a um conjunto de forças, o qual produz uma resultante constante. Dessa forma, pode-se concluir que a aceleração desse corpo também manter-se-á constante. $O$ aluno do ensino médio já está habituado a equação horário dos espaços para um movimento com aceleração constante:

$$
s(t)=s_{0}+v_{0} t+\frac{a t^{2}}{2}
$$

Uma observação mais atenta da equação 5 mostra que a mesma estará completamente definida se soubermos com antecedência os valores da posição inicial (s0) e da velocidade inicial (v0). Isso pode se tornar mais intuitivo se imaginarmos que dois corpos de mesma massa, sujeitos ao mesmo conjunto de forças, não terão necessariamente seus movimentos descritos pelas mesmas equações horárias, uma vez que os parâmetros s0 e v0 podem ser diferentes. Matematicamente falando, isso é uma consequência das condições necessárias para resolver equações diferenciais de segunda ordem, as quais exigem o conhecimento das condições iniciais para serem completamente resolvidas. 
O parágrafo anterior foi introduzido para destacar a necessidade de se explicitar a posição inicial e a velocidade inicial para se descrever a equação de qualquer movimento, no presente caso, do MHS. Portanto, ao relacionar o MHS com o MCU, deve-se conhecer de antemão a posição que o móvel inicia o MHS e, consequentemente, a respectiva posição angular no MCU associado. Também deve-se conhecer a velocidade inicial (no MHS e no MCU associado), a qual corresponde a projeção no eixo $x$ da velocidade no MCU associado. A figura 3 ilustra um MHS partindo de uma posição inicial x0, bem como a posição angular inicial $\theta 0$ do MCU associado.

Figura 3 - MHS partindo de posição inicial $x_{0}$

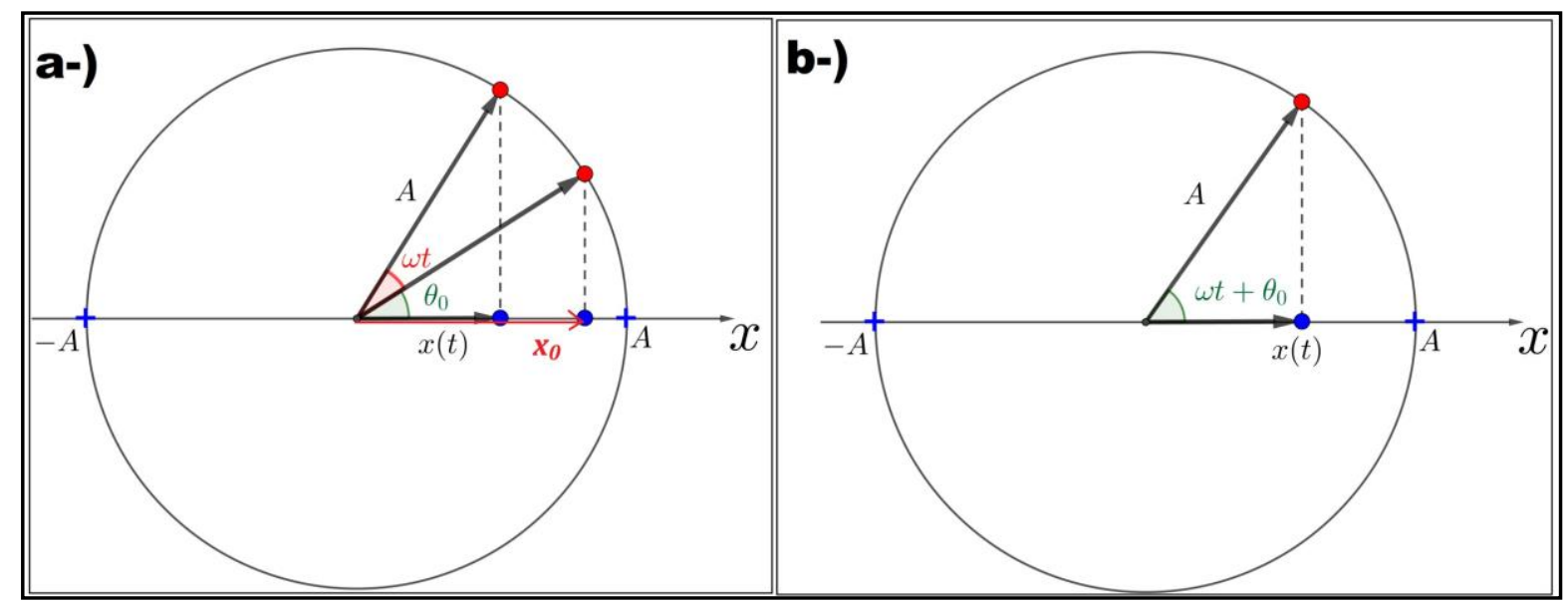

Fonte: Autores (2021)

Em que: a-) Corpo em MHS no eixo $x$. A posição $x_{0}$ está associada à posição angular $\theta_{0}$. Após um tempo $t o$ MCU associado percorre um arco $\omega t$. b-) Posição $x(t)$ no MHS corresponde a posição angular $\theta_{0}+\omega t$ no MCU associado.

Note ainda na figura 3 que passado um intervalo de tempo t, o arco descrito pelo MCU associado é wt e a posição ocupada pelo corpo em MHS é x(t).

Na parte b-) da figura, o ponto azul representa o corpo em MHS, e o ponto vermelho representa o MCU associado. Nota-se que esses pontos, juntamente com o centro da circunferência formam um triângulo retângulo. Nesse triângulo, a posição $x(t)$ é o cateto adjacente à posição angular $\omega t+\theta 0$, ou seja, $\cos \left(\omega t+\theta_{0}\right)=\frac{x(t)}{A}$. Assim:

$$
x(t)=A \cos \left(\omega t+\theta_{0}\right)
$$

Podemos utilizar um procedimento similar para determinar a equação horária da velocidade, conforme ilustrado na figura 4. 
Figura 4 - ilustração de procedimento para encontrar a equação horária da velocidade

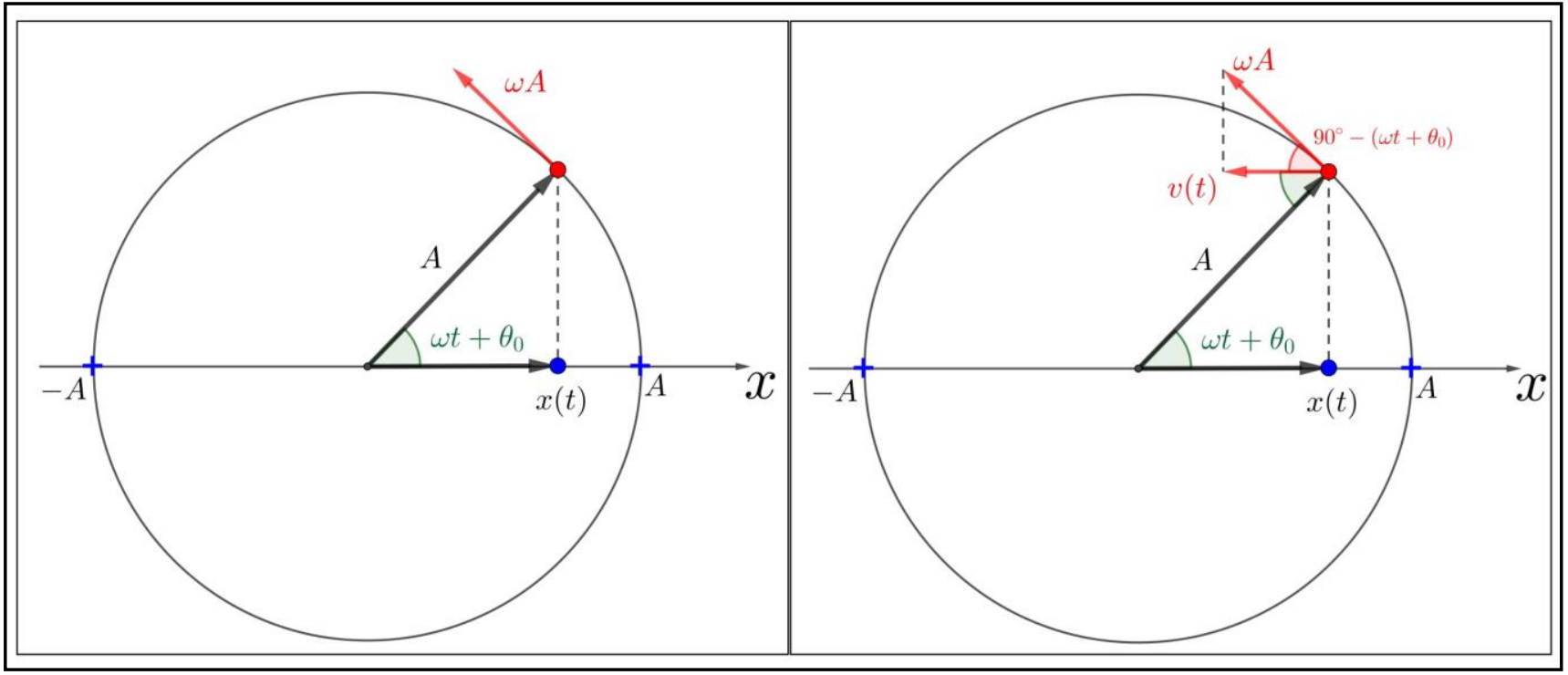

Fonte: Autores (2021)

Em que: a-) Corpo em MHS no eixo x. A velocidade $v(t)$ do MHS associada a velocidade $\vec{V}(t)$ de uma partícula em MCU. b-) Descrição dos ângulos utilizados para a obtenção da equação horária da velocidade.

Uma vez que o módulo da velocidade em qualquer MCU é constante e dada por v $=\omega R$ (onde $\mathrm{R}$ é o raio da circunferência), concluí-se que o módulo da velocidade no MCU associado ao MHS é dada por $\omega \mathrm{A}$, isto é, $|\vec{V}(t)|=\omega A$. Sabe-se que a velocidade no MCU é tangente a circunferência, ou seja, perpendicular ao raio da circunferência. Mais ainda, conforme antecipado, a velocidade do MHS (v(t)) é a projeção da velocidade do MCU ao longo do eixo $x$, situação esquematizada na parte b-) da figura 4. No ponto considerado, note que o ângulo formado entre o vetor $v(t)$ e o raio da circunferência é de $\omega t+\theta 0$, assim, o ângulo entre $\vec{V}(t)$ e o vetor $v(t)$ é $\frac{\pi}{2}-\left(\theta_{0}+\omega t\right)$. Dessa forma, o módulo da velocidade do MHS pode ser obtida por uma simples expressão trigonométrica:

$$
\cos \left[\frac{\pi}{2}-\left(\theta_{0}+\omega t\right)\right]=\frac{|v(t)|}{|\vec{V}(t)|} \Rightarrow \operatorname{sen}\left(\omega t+\theta_{0}\right)=\frac{|v(t)|}{\omega A} \Rightarrow|v(t)|=\omega A \operatorname{sen}\left(\omega t+\theta_{0}\right)
$$

Antes de prosseguir, cabe destacar que a última expressão acima foi obtida para um MHS cujo MCU associado esteja no primeiro quadrante. Sendo assim, deve-se entender como ficará essa equação para todo o movimento, mais ainda, a equação permite apenas a obtenção do módulo da velocidade. Acreditamos ser de extrema importância uma discussão acerca do movimento como um todo, isto é, uma avaliação para o movimento em todos os quadrantes. Essa discussão nos permite discutir 
fisicamente as características do MHS, bem como algumas propriedades das funções seno e cosseno. Dito isso, analisemos todos os casos possíveis.

Quando o MCU associado percorre o primeiro e o segundo quadrante, o valor de $\operatorname{sen}\left(\theta_{0}+\omega t\right)$ é positivo, ao passo que a velocidade do MHS é negativa, isto é, aponta no sentido contrário ao aumento dos valores de $x(t)$. Portanto, nessa região o sinal da velocidade do MHS é oposto ao sinal do seno. Já quando o MCU associado percorre o terceiro e o quarto quadrante, $v(t)$ é positiva, enquanto que o seno do arco associado é negativo. Concluímos que, durante todo movimento, o sinal da velocidade é oposto ao sinal do seno. consequentemente, podemos reescrever a equação $|v(t)|=\omega$ Asen $(\omega t+$ $\left.\theta_{0}\right)$, de modo a contemplar essas características:

$$
v(t)=-\omega A \operatorname{sen}\left(\omega t+\theta_{0}\right)
$$

Por fim, para encontrar a equação da aceleração em um MHS, apresenta-se a figura 5.

Figura 5 - Ilustração de como encontrar a aceleração em um MHS

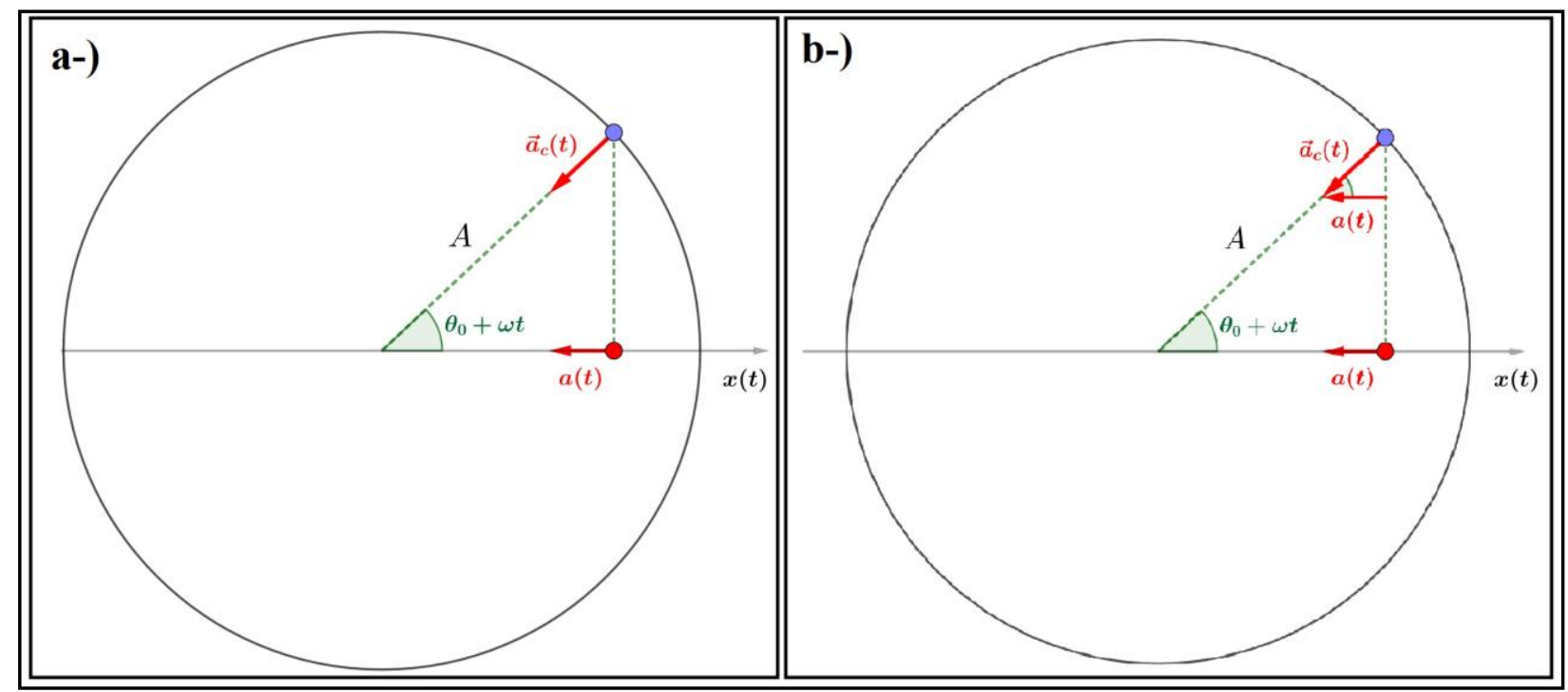

Fonte: Autores (2021)

Em que: a-) Corpo em MHS no eixo $x$. A aceleração $a(t)$ do MHS associada a aceleração centrípeta $\overrightarrow{a_{c}}(t)$ de uma partícula em MCU. b-) Descrição dos ângulos utilizados para a obtenção da equação horária da aceleração.

Assim como nos casos anteriormente discutidos, a aceleração do MHS é a projeção da aceleração da partícula que executa o MCU associado. A única aceleração presente em um MCU é a aceleração centrípeta, a qual é direcionada ao longo do raio da 
circunferência, ou seja, a aceleração $a(t)$ do MHS é a projeção no eixo $x$ da aceleração centrípeta do MCU associado (vide a parte a-) da figura 5. O ponto em MHS, o ponto que descreve o MCU associado e o centro da circunferência formam um triângulo retângulo. Por semelhança de triângulos, podemos concluir que o ângulo formado entre as extremidades dos vetores aceleração vale $\theta_{0}+\omega t$. Assim, a aceleração $a(t)$ se relaciona com aceleração centrípeta do MCU pela seguinte equação:

$$
\cos \left(\theta_{0}+\omega t\right)=\frac{|a(t)|}{\left|\vec{a}_{c}(t)\right|} \Rightarrow|a(t)|=\left|\vec{a}_{c}(t)\right| \cos \left(\theta_{0}+\omega t\right)
$$

sabemos do MCU que $\left|\vec{a}_{c}(t)\right|=\frac{|\vec{v}(t)|^{2}}{R}$, portanto:

$$
|a(t)|=\frac{|\vec{V}(t)|^{2}}{R} \cos \left(\theta_{0}+\omega t\right) \Rightarrow|a(t)|=\omega^{2} A \cos \left(\theta_{0}+\omega t\right) .
$$

Da mesma forma que feito na velocidade, devemos analisar a equação da aceleração ao longo de todo movimento, uma vez que a equação acima é válida apenas para o primeiro quadrante. Porém, aqui o trabalho é facilitado pelo fato de que a aceleração sempre terá sinal oposto ao da posição $x(t)$, assim:

$$
a(t)=-\omega^{2} A \cos \left(\theta_{0}+\omega t\right) \text { ou } a(t)=-\omega^{2} x(t) .
$$

É a partir da equação 8 que vamos obter as equações das frequências naturais de oscilação do sistema massa-mola e do pêndulo simples.

\section{ESTUDO DO SISTEMA MASSA-MOLA}

\subsection{Descrição matemática}

O estudante do ensino médio com certeza já ouviu falar na lei de Hooke. Dessa forma, antes de iniciar o estudo teórico dos trabalhos de Hooke é impotante uma breve contextualização histórica de seu referido trabalho. Robert Hooke nasceu em 1635, na Ilha de Wight ao sul da Inglaterra, quando criança mostrava muitas habilidades manuais. Aos 13 anos seu pai faleceu, fazendo com que que ele entrasse em uma escola onde aprendeu latim, grego e hebraico, inclusive conceitos da geometria euclidiana e tópicos 
de matemática. Em 1653, Hooke foi enviado para a cidade de Oxford, onde teve a oportunidade de se relacionar com diversos cientistas.

Dentre estes cientistas temos o físico e químico Robert Boyle (1627-1691), um dos fundadores da Royal Society de Londres. Boyle indicou Hooke como diretor de experimentos, o qual, com o novo cargo, tinha que utilizar suas capacidades mecânicas e inventiva na concepção de experimentos e instrumentos, além de relatá-los (ver [11]).

No ano de 1665, em resultado das suas observações microscópicas, Hooke publicou seu livro "Micrographia", no qual não somente descreve o microscópio composto por ele criado, mas também faz a descrição das suas descobertas no campo da microscopia. Após anos como diretor de experiencias, em 1677 - com a falecimento do primeiro secretário da história da Royal Society, Henry Oldenburg - Hooke assumiu o cargo mais importante da organização (ver [11]).

É importante ressaltar que Robert Hooke é principalmente conhecido pelos seus estudos com forças aplicadas a corpos deformáveis, expondo várias barras de madeiras, diversos arames e molas metálicas. As anotações e registros desses experimentos se tornaria o artigo publicado por volta de 1678, porém, para proteger seu estudo, três anos antes da publicação, fora divulgado um prospecto, ou seja, folheto usado para propaganda chamado de "Um decimo das invenções que pretendo publicar" e abaixo "a verdadeira teoria da elasticidade" junto de um anagrama em latim, "ceiiinosssttuv". Quando o artigo foi publicado, denominado de "The potentia Restitutiva, or of a Spring", revelou o significado do anagrama, com a frase do latim "ut tensio sic vis" (tal a deformação assim a força) significando que a deformação é proporcional à força aplicada.

Todavia, a referida relação linear no domínio elástico, como apresentada por Hooke, envolve a geometria do corpo ensaiado. O que realmente conhecemos como lei de Hooke esperou mais de cem anos até que, por volta de 1800, o cientista inglês Thomas YOUNG (1773-1829) mostrou a proporcionalidade não em termos de forças e alongamentos, mas em termos de tensão $(\delta)$ e extensão $(\varepsilon)$ - conceitos esses estabelecidos nessa época pelo francês Augustin CAUCHY (1789-1857). Young removeu assim os fatores geométricos e introduziu uma propriedade do material, o módulo de Elasticidade, que define a rigidez de um corpo. Consequentemente, a força aplicada é 
proporcional a deformação temos a lei de Hooke, $F=-k x$, sendo uma das leis básicas da mecânica.

O protótipo idealizado da lei de Hooke é o de uma massa preza a uma mola. Ao perturbar esse sistema, o mesmo reponde com uma força restauradora, isto é, uma força contrária ao deslocamento que a gerou. Tal força é descrita matematicamente pela lei de Hooke

$$
\vec{F}=-k \vec{x}
$$

Onde k é um número positivo relacionado à rigidez da mola, denominado a constante elástica da mola. O sinal negativo na equação 9 retrata o fato de a força $F$ ser restauradora. O vetor $\vec{x}$ é o vetor que liga a posição de equilíbrio à posição atual do corpo, sendo que o sinal negativo indica a característica restauradora da força.

Cabe ressaltar que a equação 9 pode ser utilizada para descrever qualquer MHS, necessitando apenas uma reinterpretação da constante elástica bem como do deslocamento em relação a posição de equilíbrio. Por exemplo, em ligações atômicas, é comum descrever a resultante das forças como acima, porém, naquele contexto, $k$ é um número relacionado as forças elétricas ali presentes. De qualquer modo, para pequenos deslocamentos, todas as forças restauradoras obedecem à lei de Hooke.

Figura 6 - Sistema massa-mola na horizontal

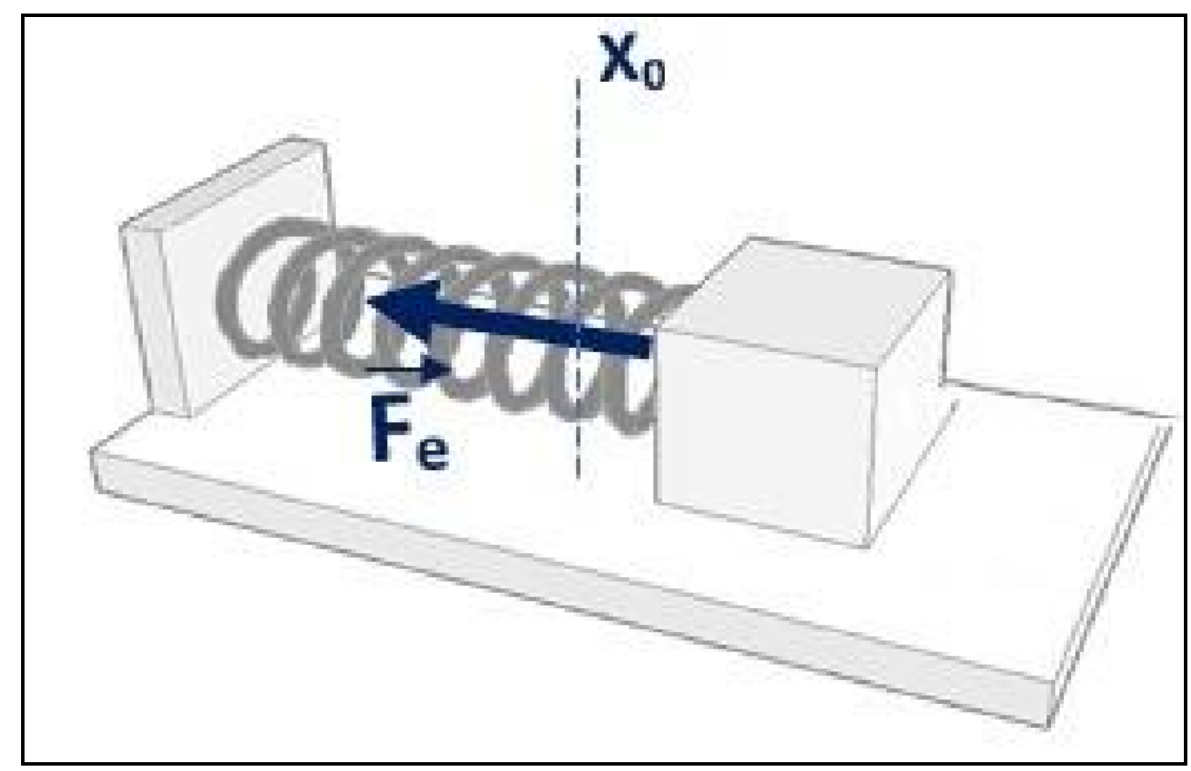

Fonte: Autores (2021) 
Destaca-se ainda que a notação vetorial pode ser omitida no caso de um MHS em apenas uma dimensão. A figura 6, ilustra a força elástica que surge quando uma massa é deslocada de sua posição de equilíbrio $x_{0}$, sendo essa a força resultante $\left(F_{r}\right)$ atuando no sistema em MHS. Partindo da segunda lei de Newton $\left(F_{r}=m a\right)$ e da equação temporal que descreve a aceleração no MHS (equação 8), tem-se:

$$
\begin{aligned}
& F_{r}=F_{\text {ela }} \Rightarrow m a(t)=-k x(t) \Rightarrow-m \omega_{m m}^{2} x(t)=-k x(t) \\
& \text { e, consequentemente, } \\
& \omega_{m m}=\sqrt{\frac{k}{m}} \text { ou } T_{m m}=\sqrt{\frac{m}{k}} .
\end{aligned}
$$

Perceba que acabamos de demonstrar a equação apresentada no início do texto sem a necessidade da introdução do conceito de equações diferenciais, mais ainda, uma vez determinado a frequência angular de oscilação, pode-se facilmente obter o período de oscilação $\left(T_{m m}\right)$. Contudo é importante destacar que implicitamente considerou-se que a posição de equilíbrio é a origem do sistema de coordenadas. Isso não é um problema em si, basta encontrar a posição de equilíbrio e considerá-la como a origem.

\subsection{Proposta de experimento: Massa-Mola}

\subsubsection{Descrição do experimento}

O propósito dessa seção é descrever uma proposta de atividade experimental que permita ao aluno visualizar a validade da equação 10. 
Figura 7 - Sistema massa-mola na vertical

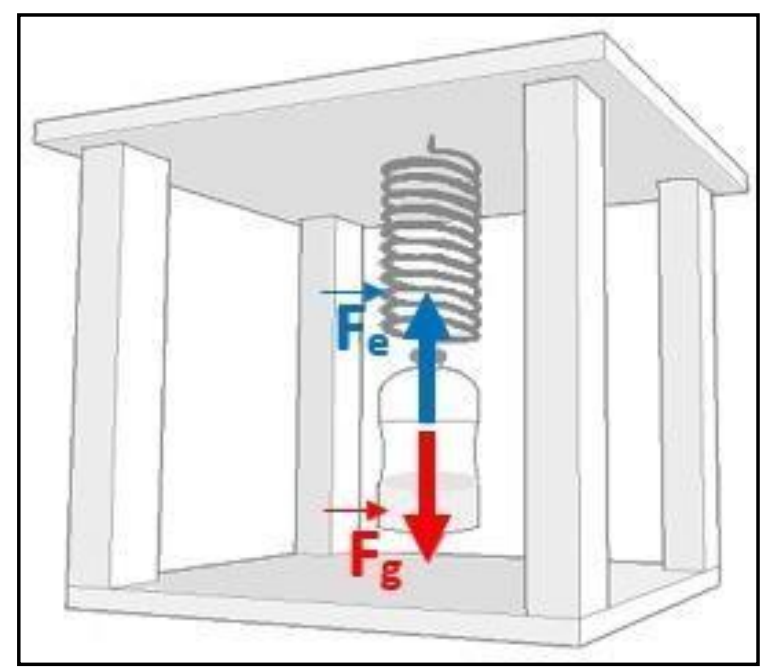

Fonte: Autores (2021)

Quando um corpo é pendurado em uma mola vertical existe uma força $F_{g}=m g$ para baixo, que é a força gravitacional, além da força elástica, Figura 7. Quando o sistema está em equilíbrio, a intensidade da força elástica é igual a intensidade da força gravitacional, isto é,

$$
m g=k x
$$

Para esse caso a posição de equilíbrio é tomada exatamente quando o sistema atinge o repouso. Dessa forma, o experimento proposto consiste em obter o valor de uma massa posta a oscilar a partir da medida do período de oscilação. Para a realização do experimento são necessários: uma garrafinha com tampa de $200 \mathrm{ml}$, um fio de arame, uma mola de caderno, água, régua, cronômetro e uma balança para comparar os resultados.

Após retirar a espiral do caderno para utiliza-la como mola, é necessário encontrar um apoio para prender a mola, como sugestão, propomos a utilização de um suporte de madeira. Em seguida, deve-se prender o arame na tampa da garrafinha que irá oscilar. A primeira parte do experimento consiste na determinação da constante elástica da mola. Para tal, mede-se com a régua o comprimento da mola relaxada, isto é, em seu estado natural. Após, enche-se a garrafinha com água até que ela atinja uma massa conhecida, com o auxílio da balança. Após, prende-se a mola com a garrafa no suporte, e então 
mede-se o quanto a mola esticou. Sendo a massa e o campo gravitacional são conhecidos (pode-se tomar 9,8m/s2), o uso da equação 11 permite a obtenção da constante elástica. Para aprimorar a medida e minimizar os erros, deve-se determinar uma constante elástica média, ou seja, deve-se repetir no mínimo três vezes o procedimento a cima, alterando a o valor da massa da garrafinha. A figura 8 ilustra o procedimento por nós adotado. Note que uma fita métrica foi colocada de modo a auxiliar a visualização do deslocamento feito pelo sistema.

Uma vez determinado o valor médio de k, inicia-se a segunda parte do experimento, a qual consiste em determinar uma quantidade de massa desconhecida e compará-la com a medida dessa mesma massa feita por uma balança. Dessa forma, prende-se esta nova massa a mola, espera-se a obtenção do equilíbrio e então deslocase a levemente o sistema de sua posição de equilíbrio, fazendo-o oscilar.

Figura 8 - Esquema experimental utilizado para obtenção de uma massa desconhecida

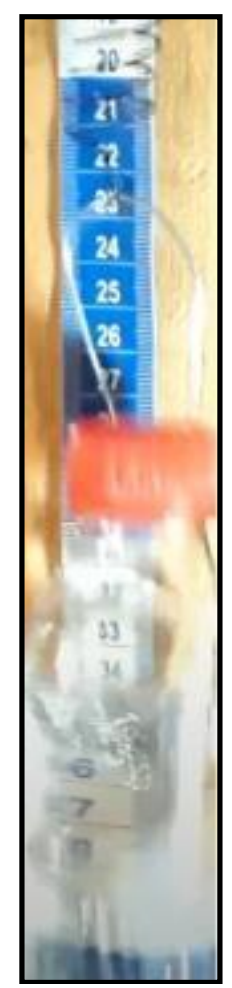

Fonte: Autores (2021)

Utilizando o cronômetro, determina-se o tempo de vinte oscilações, de modo a encontrar uma boa média para o tempo de uma única oscilação. A etapa acima foi repetida por dez vezes. De posse do conjunto de dados anotados, substitui-se o valor do 
tempo de uma oscilação e da constante k, calculada na etapa anterior na equação 10 , obtendo assim o valor da massa. Após, compara-se o valor da massa calculado ao valor obtido pela medida direta em uma balança.

\subsubsection{Resultados obtidos}

Para obtenção da constante elástica da mola, foram utilizados as seguintes massas: $50 \mathrm{~g}, 100 \mathrm{~g}$ e $150 \mathrm{~g}$. As deformações obtidas, bem como os valores calculados de $k$ estão listadas na tabela 1.

Tabela 1 - Deslocamentos obtidos na determinação de $k$

\begin{tabular}{|c|c|c|}
\hline Massa $(g)$ & Deslocamento $(\mathrm{cm})$ & $K(N / m)$ \\
\hline 50 & 1 & 49 \\
\hline 100 & 2 & 49 \\
\hline 150 & 3 & 49 \\
\hline
\end{tabular}

Fonte: Autores (2021)

Os valores do tempo necessário para a realização de 20 oscilações estão listados na tabela 2.

Tabela 2 - Tempo medido para 20 oscilações

\begin{tabular}{|c|c|}
\hline$n$ & $T(s)$ \\
\hline 1 & 8,97 \\
\hline 2 & 8,98 \\
\hline 3 & 9,01 \\
\hline 4 & 8,82 \\
\hline 5 & 8,82 \\
\hline 6 & 8,84 \\
\hline 7 & 8,70 \\
\hline 8 & 8,67 \\
\hline 9 & 8,85 \\
\hline 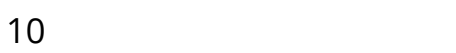 & 8,89 \\
\hline
\end{tabular}

Fonte: Autores (2021)

Um cálculo simples indica que o tempo médio para a realização de 20 oscilações é de $8,855 \mathrm{~s}$, isto é, uma única oscilação tem um período $T_{m m}$ de $0,443 \mathrm{~s}$. Isolando a massa da equação 10 e substituindo os valores aqui encontrados, tem-se: 


$$
m=k\left(\frac{T_{m m}}{2 \pi}\right) \Rightarrow m=49\left(\frac{0,443}{2 \pi}\right) \Rightarrow m=0,2433 g .
$$

Para comparar o resultado, utilizou-se a balança, cujo resultado apresentado foi de $240 \mathrm{~g}$ (vide figura 9). Aqui destacamos que o propósito desse experimento não é obter um valor fidedigno da massa, mas sim apresentar aos alunos um experimento qualitativo que os permita compreender de maneira concreta a validade da teoria discutida previamente.

Figura 9 - Medida da massa desconhecida

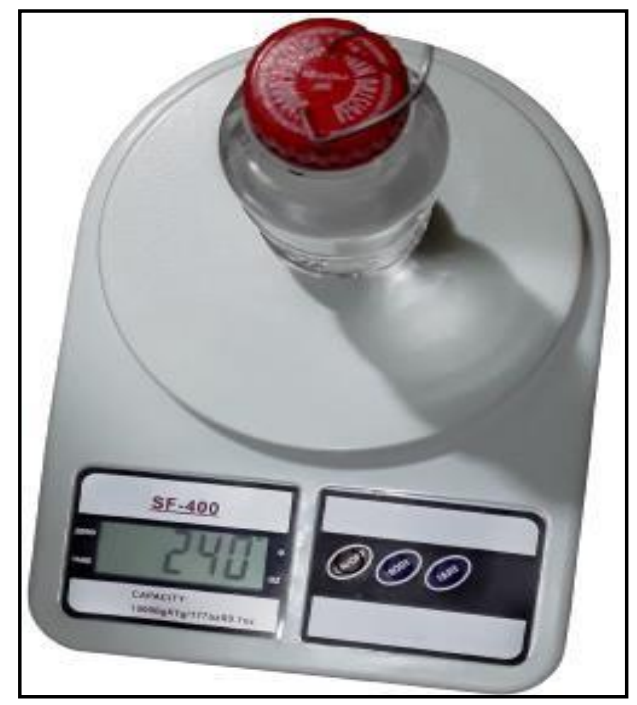

Fonte: Autores (2021)

\section{PROPOSTA DE EXPERIMENTO: PÊNDULO SIMPLES}

\subsection{Descrição matemática}

Galileu Galilei sempre foi muito dedicado ao estudo do movimento dos corpos, sendo um dos primeiros estudiosos a refletir sobre o movimento oscilatório. Diz a história que uma vez Galileu estava na catedral de Pisa quando começou a olhar e refletir sobre o movimento que um lustre fazia no alto da igreja e teve a ideia de medir o tempo que aquele lustre demorava para oscilar. Como naquela época não existia relógio e nem cronômetro, Galileu mediu o tempo das oscilações com as batidas de seu pulso. O que despertou a atenção de Galileu foi que a amplitude de oscilação desse sistema parecia não alterar o período de oscilação do mesmo [1]. 
O fato observado por Galileu e descrito no parágrafo anterior pode ser compreendido ao se estudar o movimento de um pêndulo simples. Um pêndulo simples é um sistema formado por um objeto de massa m preso a um fio vertical inextensível de comprimento $\ell$. Na posição vertical o sistema se encontra em equilíbrio, porém, ao tirar o pêndulo dessa posição e soltá-lo ele executará um movimento oscilatório. A figura 10 ilustra o pêndulo simples, bem como as forças responsáveis pelo movimento oscilatório.

Figura 10 - Ilustração de pêndulo simples e forças responsáveis pelo movimento oscilatório

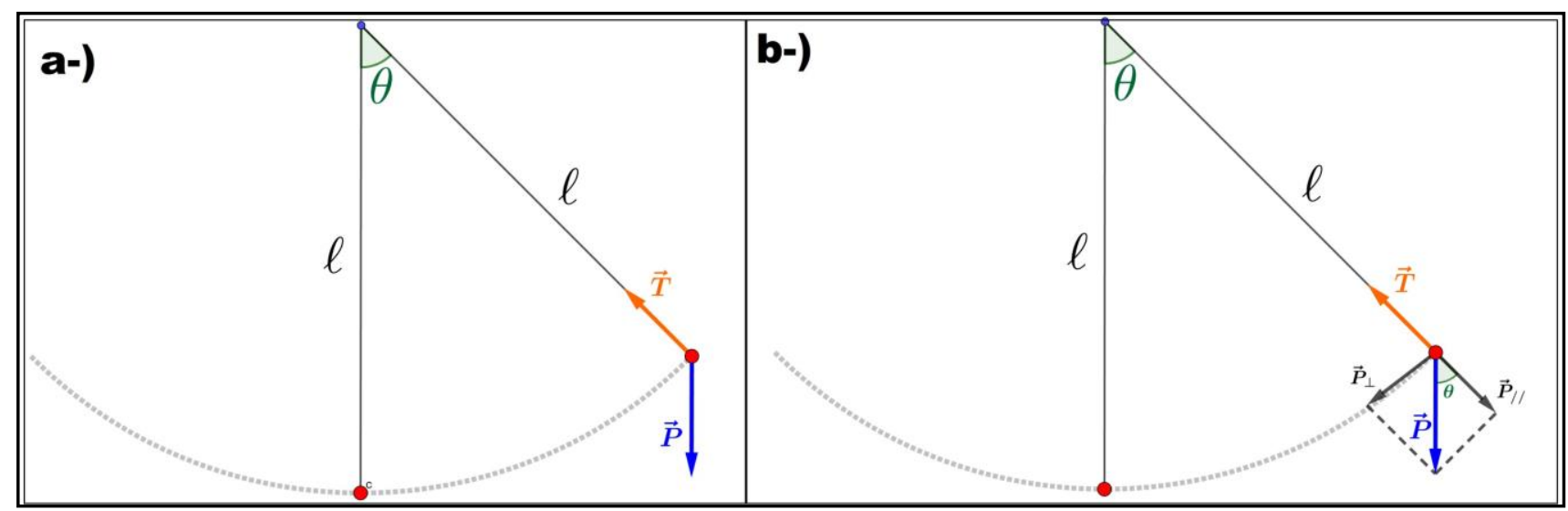

Fonte: Autores (2021)

Em que: a-) O sistema foi retirado da posição de equilíbrio, causando assim um desequilíbrio entre as forças peso $\vec{P}$ e tração $\vec{T}$. b-) Como não há movimento na direção do fio obviamente a força peso deve ser decomposta, sendo $\vec{P}_{/ /}$a componente paralela à tração e $\overrightarrow{P_{\perp}}$ a componente perpendicular à tração $\vec{T}$.

Partindo da figura percebe-se que força resultante é a componente $\vec{P}_{\perp}$ da força peso. Nota-se ainda que a resultante é uma força restauradora, ou seja, aponta para a posição de equilíbrio. Nesse caso, tem-se um movimento bi-dimensional, entretanto, esse movimento pode ser completamente descrito pela variável $\theta$, o ângulo entre a posição do fio e a vertical. Dessa forma, tem-se um movimento que depende de apenas uma variável. Por questão de simplicidade considera-se $\theta$ como sendo positivo quando localizado a direita da posição de equilíbrio (conforme mostrado na figura 10), e negativo quando o pêndulo se encontrar no lado esquerdo da posição de equilíbrio.

Seguindo todas as considerações até aqui apontadas e o fato geométrico $\left|\vec{P}_{\perp}\right|=$ $|\vec{P}| \operatorname{sen}(\theta)$, tem-se: 


$$
\vec{F}_{r}=\vec{P}_{\perp} \Rightarrow\left|\vec{F}_{r}\right|=\left|\vec{P}_{\perp}\right| \Rightarrow m|a(t)|=m|g \operatorname{sen}(\theta)| \Rightarrow|a(t)|=|g \operatorname{sen}(\theta)|
$$

Para tornar o sistema mais simples (e sermos fieis as observações feitas por Galileu) analisaremos esse sistema considerando apenas pequenos deslocamentos angulares, isto é, para valores de $\theta$ próximos de 0. Dessa forma, como o próprio aluno pode verificar com uma calculadora científica, tem-se a relação $\operatorname{sen}(\theta) \approx \theta$, ou seja, $|a(t)|=|g \theta|$. Com essa aproximação e as considerações feitas acerca do sinal de $\theta$, notamos que a aceleração é sempre no sentido oposto ao de $\theta$, assim,

$$
a(t)=-g \theta(t) \Rightarrow-\omega^{2} \theta(t)=-g \theta(t)
$$

De posse da equação anterior, obtém-se a equação do período de um pêndulo simples:

$$
\omega_{p s}=\sqrt{\frac{g}{\ell}} \text { ou } T_{p s}=2 \pi \sqrt{\frac{\ell}{g}}
$$

\section{PROPOSTA DE EXPERIMENTO: PÊNDULO SIMPLES}

\subsection{Descrição do experimento}

Conforme antecipado, o presente experimento tem por objetivo a determinação da aceleração gravitacional local a partir da equação 12.

Com esse propósito em mente, montou-se um sistema constituído de uma pequena bolinha de gude presa um fio (supostamente inextensível) de 0,6m de comprimento por uma cola quente. Cabe destacar que quanto maior for o fio, mais confiáveis serão os dados obtidos. Essa confiabilidade se deve ao fato de que um fio de maior comprimento aumenta o período de oscilação (facilitando a medida do mesmo), além de possibilitar que utilizemos desvios bem "pequenos" da posição de equilíbrio, melhorando muito a aproximação $\operatorname{sen}(\theta) \approx \theta$. 
Note que a equação 12 não contém a massa, ou seja, não é necessário estipular a massa da bolinha de gude, basta apenas garantir que os efeitos do ar serão desprezíveis. O sistema por nós utilizado se encontra na figura 11.

Figura 11 - Ilustração do protótipo utilizado

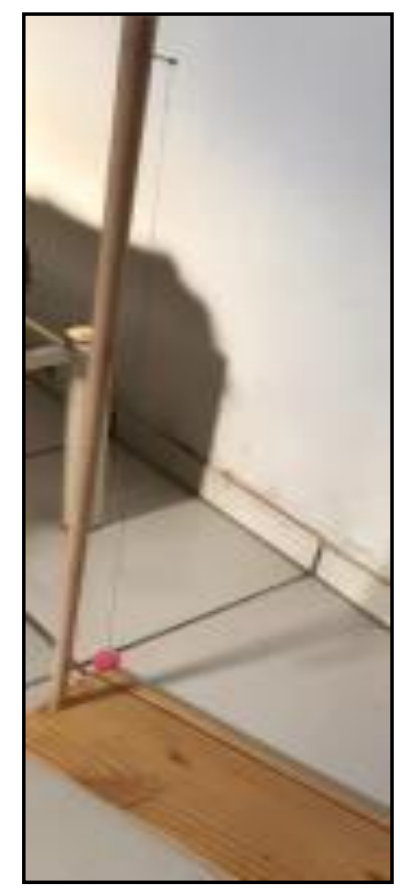

Fonte: Autores (2021)

Uma vez montado o experimento, o pêndulo foi levemente deslocado de sua posição de equilíbrio (de modo a obtermos um pequeno ângulo de deslocamento) e posto a oscilar, então, com o auxílio de um cronômetro, media-se o tempo de 10 oscilações. Com a média do tempo dessas 10 oscilações, obtém-se o período de uma única oscilação. Uma vez conhecido o valor do período de oscilação e do comprimento do fio, utiliza-se a equação 12 e calcula-se o valor da aceleração gravitacional local.

\subsection{Resultados Obtidos}

Conforme indicado, o comprimento do fio utilizado foi de 0,6m. Cabe destacar que o experimento pode ser refeito com diferentes comprimentos, minimizando assim possíveis erros. Foram feitos 10 medidas de tempo, e os dados obtidos se encontram na tabela 3. 
Tabela 3 - Tempo medido para 20 oscilações

\begin{tabular}{|c|c|}
\hline Medida & $T$ (10 Oscilações) (s) \\
\hline 1 & 15,94 \\
\hline 2 & 16,02 \\
\hline 3 & 15,91 \\
\hline 4 & 15,99 \\
\hline 5 & 15,77 \\
\hline 6 & 15,79 \\
\hline 7 & 15,79 \\
\hline 8 & 15,82 \\
\hline 9 & 15,93 \\
\hline 10 & 16,03 \\
\hline
\end{tabular}

Fonte: Autores (2021)

Assim, tomamos a média aritmética dos valores presentes na tabela 3 como sendo o período de 10 oscilações, no qual obtivemos o valor 15,89. Dessa forma, o período de uma única oscilação é $T_{p s}=\frac{15,89}{10}=1,58 s$. Substituindo esse valor na equação do período (equação 12) e isolando a aceleração gravitacional, tem-se:

$$
g=\ell\left(\frac{2 \pi}{T_{p s}}\right) \Rightarrow g=0,6\left(\frac{2 \pi}{1,58}\right) \Rightarrow g=9,51 \mathrm{~ms} / \mathrm{s}^{2}
$$

\section{CONCLUSÃO}

Destacamos aqui que o propósito do trabalho não era obter um valor preciso do campo gravitacional, nem da massa desconhecida. O propósito é apresentar uma proposta de abordagem teórica e experimental do conceito de MHS ao aluno do ensino médio. Ao longo do texto ainda apresentaram-se algumas considerações históricas, as quais devem ser incluídas como um agente facilitador do ensino de física (ver [7]).

Entendemos que abordagens que apenas apresentam as equações em seu formato final, sem uma profunda discussão do processo de como obtê-las, não contribui para despertar a curiosidade e nem motivam o aluno ao estudo de física. Com esse intuito o trabalho foi desenvolvido e uma proposta de experimento foi posta em prática. Também acreditamos que a abordagem aqui apresentada faz com que o aluno tenha que compreender melhor o movimento circular uniforme, bem como os conceitos ali 
envolvidos: período e frequência, uma vez que a demonstração das equações do MHS, bem como as atividade experimentais propostas exigem uma compreensão desses conceitos.

Por fim, destacamos a necessidade de se buscar novas abordagens para o ensino de determinados conteúdos de física no ensino médio, de modo a sempre apresentar a construção teórica desses conceitos, uma vez que a simples matematização e exposição das equações finais pouco, ou em nada, contribuem para a formação dos alunos. Esperamos com esse trabalho oferecer ao professor do ensino médio uma proposta de abordagem, a qual pode ser adequadas à realidade de cada contexto, de modo a auxiliar no ensino desse conteúdo tão abstrato e importante.

\section{REFERÊNCIAS}

Eves, H. W. (1995). Introdução à história da matemática. Unicamp.

Ferreira, A. L., Borrero, P. P. G. (2005). Uma proposta para o ensino de oscilações. RECEN-Revista Ciências Exatas e Naturais, 7(2), 155-171.

Ferreira, D. B., Villani, A. (2002). Uma reflexão sobre prática e ações na formação de professores para o ensino de física. Revista Brasileira de Pesquisa em Educação em Ciências, 2.

Feynman, R. P., Leighton, R. B., Sands, M. (2008). Lições de Física - Vol. 1. Bookman.

Hewitt, P. G. (2007). Fundamentos de física conceitual, $9^{\circ}$ edn. Bookman.

Neto, A. P. S., dos Santos, A. B. (2015). Atividade para ensino de oscilações e ondas no ensino fundamental.

Neves, M. C. D. (1998). A história da ciência no ensino de física. Ciência \& Educação (Bauru), 5(1), 73-81.

Nussenzveig, H. M. (2002). Curso de Física Básica: fluidos, oscilações e ondas, calor, $4^{\circ}$ edn. Editora Blucher.

Pinheiro, D. M., Murakami, G. E., Beraldo, N., Witkowski, F. M., Brunelli, D. D., Germano, J. S. E. (2013). Uso do software de simulação interactive physics como ferramenta de apoio ao professor em sala de aula no ensino de oscilações mecânicas. Em: CONGRESSO BRASILEIRO DE EDUCAÇÃO EM ENGENHARIA.

Pinto, S., Silva, L., Tenório-de Carvalho, C., Egoavil, C. (2004). Pêndulo simples utilizando tecnologia embarcada de baixo custo aplicado ao ensino da física. Lat Am J Sci Educ, 2015, 2-6. 
ROONEY, A. (2013). A História da Física: da Filosofia ao Enigma da Matéria Negra, $1^{\circ}$ edn. São Paulo-M. Books do Brasil Editora Ltda.

Young, H. D., Freedman, R. A. (2015). Física, II: Termodinâmica e ondas, $14^{\circ}$ edn. Bookman.

\section{Contribuições de autoria}

\section{1 - Rafaela Coutinho de Oliveira}

Discente do curso de Licenciatura em Física

https://orcid.org/0000-0003-0065-4138 - rafaelacoutinho98@gmail.com

Contribuições: Conceituação, Curadoria dos dados, Escrita - primeira redação

\section{2 - Édino da Silva Brito}

Discente do curso de Licenciatura em Física https://orcid.org/0000-0001-9991-1979 - edino.brito@aluno.ifsp.edu.br Contribuições: Conceituação, Curadoria dos dados, Escrita - primeira redação

\section{3 - Gabriel Willian Callegari}

Discente do curso de Licenciatura em Física https://orcid.org/0000-0002-3452-9119 - loumuck@outlook.com Contribuições: Conceituação, Curadoria dos dados, Escrita - primeira redação

\section{4 - Alexandre Jitsuo Fuzita}

Discente do curso de Licenciatura em Física https://orcid.org/0000-0001-7445-8047 - fuzitaalexandre@gmail.com Contribuições: Conceituação, Curadoria dos dados, Escrita - primeira redação

\section{5 - Vitor Hugo Dias Ferrara}

Discente do curso de Licenciatura em Física https://orcid.org/0000-0003-4011-8014 - vitor-hdf@hotmail.com Contribuições: Conceituação, Curadoria dos dados, Escrita - primeira redação

\section{6 - Daniela Lopes Elizeu}

Discente de Licenciatura em Física https://orcid.org/0000-0002-7675-9473 - danielalopesifsp@gmail.com Contribuições: Conceituação, Curadoria dos dados, Escrita - primeira redação

\section{7 - Saulo Portes dos Reis}

Doutor em Ciência dos Materiais https://orcid.org/0000-0002-9327-403X - spdreis@ifsp.edu.br

Contribuições: Conceituação, Escrita - revisão e edição

\section{Como citar este artigo}

OLIVEIRA, R. C.; BRITO, E. S.; CALLEGARI, G. W.; FUZITA, A. J.; FERRARA, V. H. D.; ELIZEU; D. L.; REIS, S. P. MHS e MCU: a conexão desses movimentos no ensino de física. Ciência e Natura, Santa Maria, v. 43, e79, p. 1-28, 2021. Disponível em: https://doi.org/10.5902/2179460X64852. 